\title{
Machine Perfusion in Liver Transplantation: A Systematic Review and Meta-Analysis
}

\author{
Matas Jakubauskas ${ }^{a, b}$ Lina Jakubauskiene ${ }^{a, b}$ Bettina Leber ${ }^{a}$ \\ Kestutis Strupas ${ }^{\mathrm{b}}$ Philipp Stiegler ${ }^{\mathrm{a}}$ Peter Schemmer ${ }^{\mathrm{a}}$ \\ ${ }^{a}$ General, Visceral and Transplant Surgery, Department of Surgery, Medical University of Graz, Graz, Austria; \\ ${ }^{b}$ Faculty of Medicine, Vilnius University, Vilnius, Lithuania
}

\section{Keywords}

Machine perfusion - Liver transplantation - Extended criteria donors $\cdot$ Marginal grafts

\footnotetext{
Abstract

Background: Liver transplantation (LTx) is the only treatment option for patients with end-stage liver disease. Novel organ preservation techniques such as hypothermic machine perfusion (HMP) or normothermic machine perfusion (NMP) are under investigation in order to improve organ quality from extended criteria donors and donors after circulatory death. The aim of this study was to systematically review the literature reporting LTx outcomes using NMP or HMP compared to static cold storage (SCS). Methods: The following data were retrieved: graft primary nonfunction rate, early allograft dysfunction (EAD) rate, biliary complication rate, and 12-month graft and patient survival. A total of 15 studies were included (6 NMP and 9 HMP studies), and meta-analysis was performed only for HMP studies because NMP had considerable differences. Results: The systematic review showed the potential of NMP to reduce graft injury and lower the liver graft discard rate. The performed quantitative analyses showed that the use of HMP reduces the rate of EAD (odds ratio [OR] 0.51;95\% confidence interval $\left.[\mathrm{Cl}] 0.34-0.76 ; p=0.001 ; R^{2}=0 \%\right)$ and nonanastomotic biliary strictures (OR $0.34 ; 95 \% \mathrm{Cl} 0.17-0.67 ; p=$ $0.002 ; P^{2}=0 \%$ ) compared to SCS. Conclusion: Our systematic review and meta-analysis revealed that the use of HMP reduces the rate of EAD and non-anastomotic biliary strictures compared to SCS.

\section{Introduction}

Several decades since the first attempt in 1963, liver transplantation (LTx) remains the only treatment option for patients with end-stage liver disease [1]. Although advances in surgical technique, immunosuppression, and organ preservation led to greatly improved postoperative outcomes, the steadily increasing organ demand is unmet, resulting in organ shortage worldwide [2]. According to the Organ Procurement and Transplantation Network data, in 2019, in the USA, there were 8,896 LTx opposing 13,448 candidates newly added to the waiting list in the same year. Furthermore, 2,415 patients became too sick to be transplanted or died while waiting for LTx. In the Eurotransplant network countries, 1,687 LTx were performed in 2019; however, the waiting list increased by 2,502 new registrations. The discrepancy between the need and availability of liver grafts requires expanding the donor pool with both extended criteria donors (ECDs) and donors after circulatory death (DCD). Therefore, the development of novel organ preservation techniques is mandatory in order to increase the donor organ pool.

For the last few decades, static cold storage (SCS) remained the basically unchanged gold standard in preserving high-quality organs due to its low cost and simplicity [3]. However, its limitations in expanding the donor pool by including ECD organs are well known. Vogel et al. [4]

Lina Jakubauskiene and Matas Jakubauskas contributed equally to this article. mercial purposes requires written permission. 


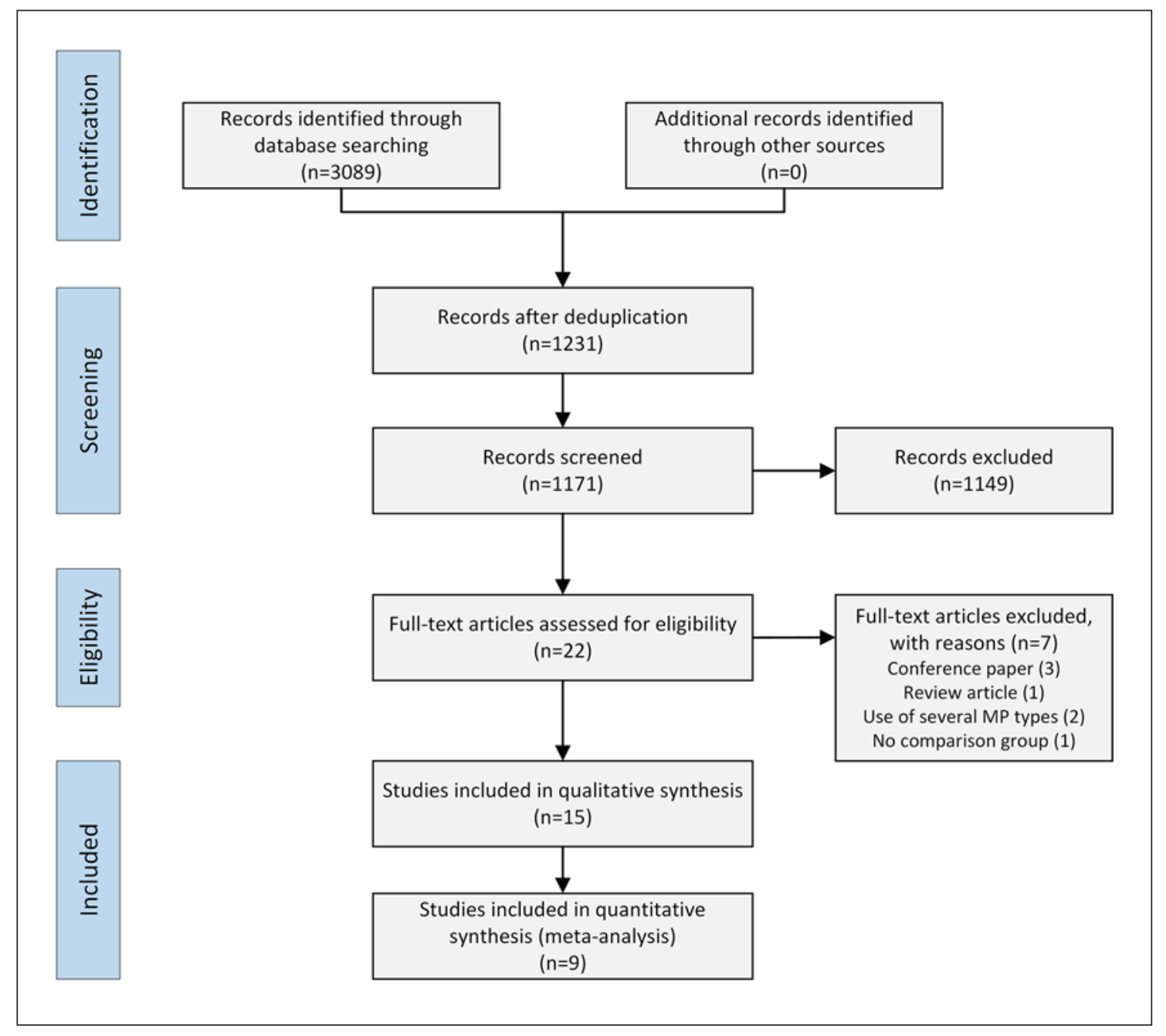

Fig. 1. PRISMA flowchart of study selection process. MP, machine perfusion.

outlines 4 major weaknesses of SCS: (I) sustained organ injury is not reversed; (II) further organ injury during storage continues; (III) organ viability cannot be assessed; and (IV) storage time is limited. Some of these shortcomings can be overcome by utilizing machine perfusion (MP). Several modes of MP are possible differing in temperature, perfusion device, perfusion solution, etc. So far, in a clinical setting, the 2 most studied types of MP are hypothermic machine perfusion (HMP) and normothermic machine perfusion (NMP) [5]. HMP relies on the reduced cell metabolism in hypothermic conditions, additionally washing out toxins accumulated during storage [6]. NMP takes a different approach by sustaining the full cell metabolism at body temperature, allowing organ viability assessment before transplantation [7]. However, the high cost and nonconclusive evidence limits its wider use in the LTx setting. The aim of this study was to systematically review the literature reporting LTx outcomes when using NMP or HMP for organ preservation compared to SCS.

\section{Methods}

Literature Search Strategy

No ethics approval was required for this type of study. Literature search was performed in PubMed, Web of Science, and EMBASE databases. The following combination of Medical Subject Headings (MeSH) and keywords with the employment of " $A N D$ " or "OR" Boolean operators were used: "Liver" OR "Liver Transplantation" AND "Machine perfusion" OR "Hypothermic perfusion" OR "Subnormothermic perfusion" OR "Normothermic perfusion."

The search was restricted to English language only, without a time limitation. The most recent search was performed on May 19, 2021. Database-specific search strategies are provided as online supplementary material (for all online suppl. material, see www. karger.com/doi/10.1159/000519788).

\section{Eligibility Criteria}

We included studies that compared the use of NMP or HMP with SCS in an LTx setting. According to Karangwa et al. [8] standardized nomenclature proposal cutoff values of $>35^{\circ} \mathrm{C}$ for NMP and $<12^{\circ} \mathrm{C}$ for HMP were used when including studies. Randomized controlled trials (RCTs), cohort studies, case-control studies, and quasi-randomized studies were eligible for inclusion. Case re- 


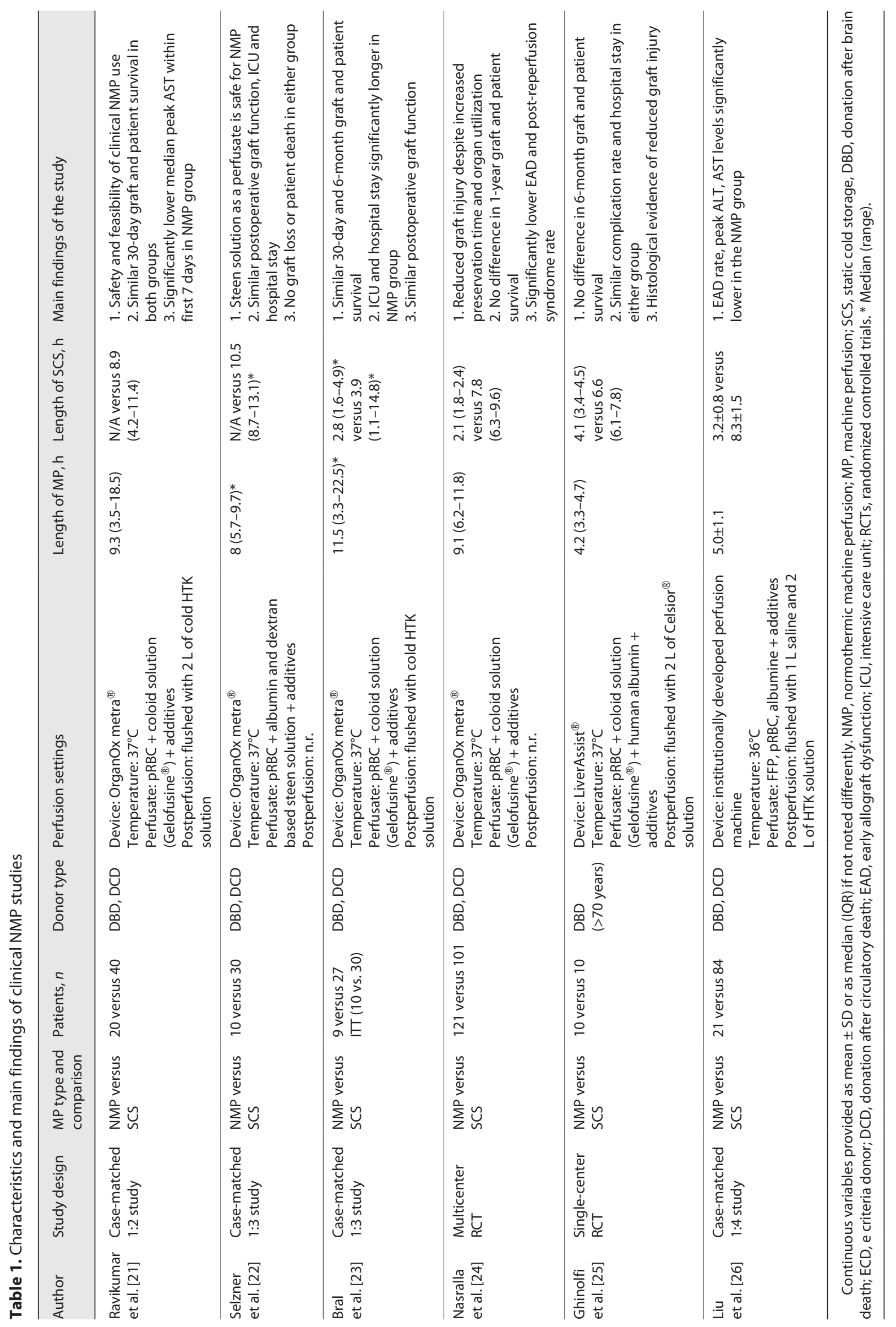




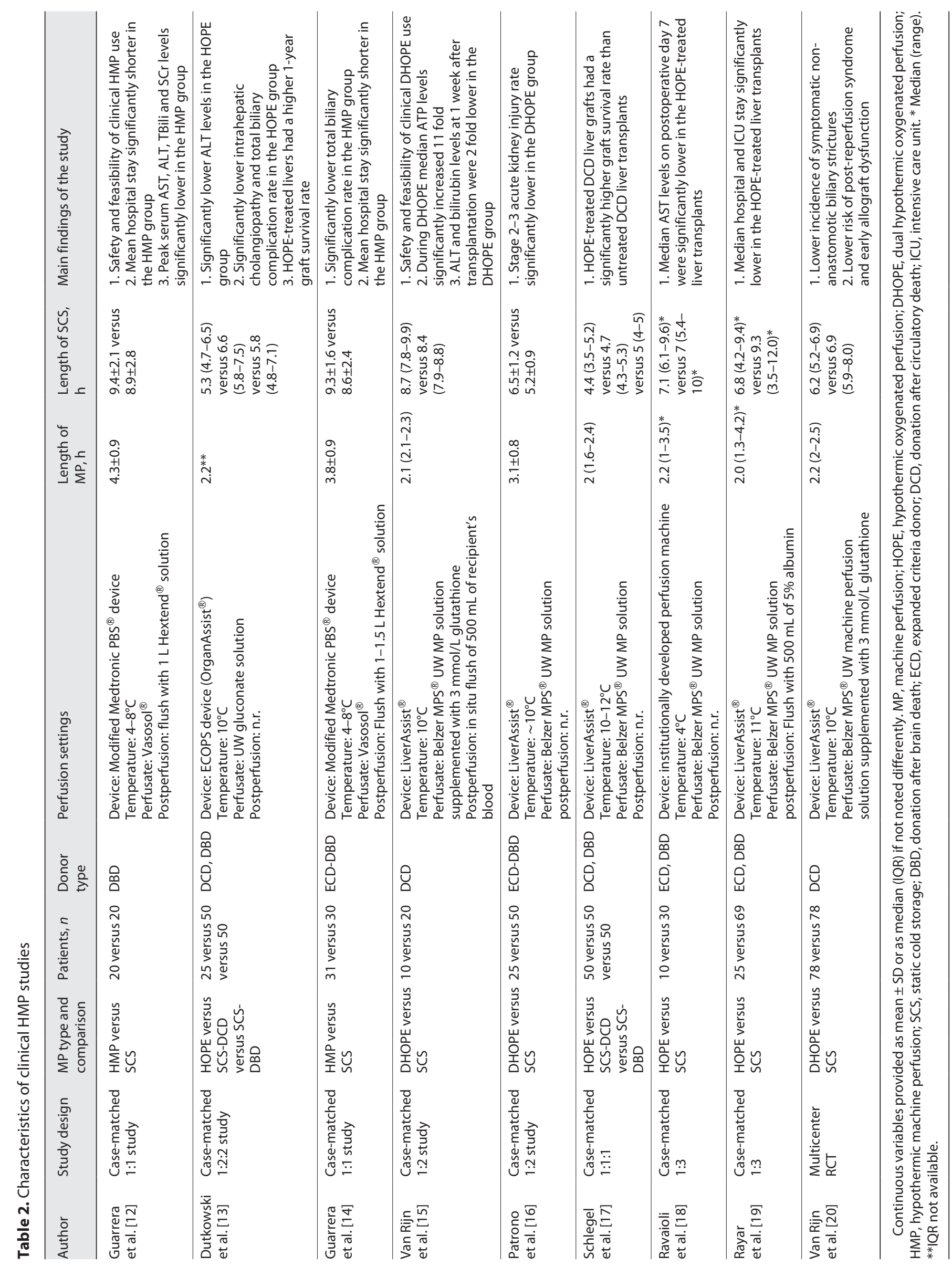


Table 3. Risk of bias assessment of included nonrandomized studies using the ROBINS-I tool

\begin{tabular}{llllllll}
\hline & Confounding & $\begin{array}{l}\text { Selection of } \\
\text { participants }\end{array}$ & $\begin{array}{l}\text { Classification } \\
\text { of inter- } \\
\text { ventions }\end{array}$ & $\begin{array}{l}\text { Deviation from } \\
\text { intended } \\
\text { intervention }\end{array}$ & Missing & $\begin{array}{l}\text { Measure- } \\
\text { data }\end{array}$ & $\begin{array}{l}\text { Selection of } \\
\text { ment of reported } \\
\text { outcomes }\end{array}$ \\
results
\end{tabular}

NMP, normothermic machine perfusion; HMP, hypothermic machine perfusion.

ports, case series (sample size less than 10 patients), and studies including children or animals were excluded.

\section{Study Selection and Data Extraction}

At first, the studies were screened based on their title and abstract. Full text was obtained for potentially eligible studies. The following data were extracted from all included studies: study characteristics, year of publication, sample size, donor type, MP parameters, and organ preservation length. For the outcome assessment, additional data were obtained: graft primary nonfunction (PNF) rate, early allograft dysfunction (EAD) rate, biliary complication rate, and 12-month graft and patient survival.

\section{Risk of Bias Assessment}

The quality of included nonrandomized studies was evaluated using the ROBINS-I risk of bias assessment tool [9]. Additionally, the quality of included RCTs was evaluated using the RoB 2 risk of bias assessment tool [10].

\section{Statistical Analysis}

We performed the meta-analyses using the software package RevMan 5.4.1 according to the recommendations of The Cochrane Handbook for Systematic Reviews and Interventions [11]. When analyzing HMP studies, we further subdivided them into 2 groups, ones that used additional oxygen during MP and ones that did not. For dichotomous variables, we calculated odd ratios (ORs) with $95 \%$ confidence interval (CI). As we expected a high level of heterogeneity across studies, Mantel-Haenszel (M-H) method and randomeffects models were employed. Furthermore, the $I^{2}$ test was used to measure statistical heterogeneity. If a study observed no event in either group, it was not included in the quantitative analysis.

\section{Results}

\section{Study Selection and Characteristics}

Literature search results and the selection process of the studies are presented in the PRISMA flowchart
(Fig. 1.). The initial search retrieved 3,089 potentially relevant studies. After evaluating 22 full-text articles, 15 of them were included in the qualitative synthesis [12-26]. Due to high heterogeneity between studies analyzing $\operatorname{NMP}(n=6)$, only studies investigating $\operatorname{HMP}(n=9)$ were included in the meta-analysis. Main characteristics of studies examining NMP and HMP are presented in Tables 1 and 2 respectively. In 2 studies, we recognized overlapping patient cohorts; therefore, we mainly extracted outcome data from the lately published study, which has a larger sample size $[13,17]$. Unfortunately, this study did not report the $\mathrm{EAD}$ rate, and after failure in contacting the authors, we decided to extract the EAD rate from their first study. Additionally, when evaluating these studies as a control group for the meta-analysis, we included untreated DCD liver transplant data.

\section{Study Quality}

All included nonrandomized studies, except one, showed moderate risk of bias (Table 3). The intervention domain in studies by Dutkowski et al. [13] and Schlegel et al. [17] was evaluated as having serious risk of bias due to differences of immunosuppression therapy between the HMP and SCS groups. These cohorts were included from different transplant centers; immunosuppression differed between the groups, and this may have affected the outcomes. The methodological quality of the 3 included randomized studies is summarized in Table 4.

\section{Outcome Assessment}

Normothermic Machine Perfusion

A total of 6 studies analyzed the effect of NMP in LTx (Table 1) [21-26]. Nasralla et al. [24] conducted the 
Table 4. Risk of bias assessment of included randomized studies using the ROBINS 2 tool

\begin{tabular}{|c|c|c|c|c|c|c|}
\hline & $\begin{array}{l}\text { Randomization } \\
\text { process }\end{array}$ & $\begin{array}{l}\text { Deviations from } \\
\text { intended intervention }\end{array}$ & $\begin{array}{l}\text { Missing } \\
\text { outcome data }\end{array}$ & $\begin{array}{l}\text { Measurement } \\
\text { of the outcome }\end{array}$ & $\begin{array}{l}\text { Selection of the } \\
\text { reported result }\end{array}$ & $\begin{array}{l}\text { Overall risk of bias } \\
\text { judgment }\end{array}$ \\
\hline \multicolumn{7}{|l|}{ NMP studies } \\
\hline Nasralla et al. [24] & Low & Some concerns & Low & Low & Low & Some concerns \\
\hline Ghinolfi et al. [25] & Low & Some concerns & Low & Some concerns & Some concerns & Some concerns \\
\hline \multicolumn{7}{|l|}{ HMP studies } \\
\hline Van Rijn et al. [20] & Low & Some concerns & Low & Low & Low & Some concerns \\
\hline
\end{tabular}

NMP, normothermic machine perfusion; HMP, hypothermic machine perfusion.

\begin{tabular}{|c|c|c|c|c|c|c|c|c|c|c|}
\hline \multirow{2}{*}{$\begin{array}{l}\text { Study or Subgroup } \\
1.1 .1 \text { Oxygated }\end{array}$} & \multirow[t]{2}{*}{$\begin{array}{l}\text { HMP } \\
\text { Events }\end{array}$} & \multirow[t]{2}{*}{ Total } & \multicolumn{2}{|c|}{ SCS } & \multirow[t]{2}{*}{ Weight } & \multirow[t]{2}{*}{$\begin{array}{l}\text { Odds Ratio } \\
\text { M-H, Random, } 95 \% \mathrm{CI}\end{array}$} & \multicolumn{4}{|c|}{$\begin{array}{c}\text { Odds Ratio } \\
\mathrm{M}-\mathrm{H}, \text { Random, } 95 \% \mathrm{Cl}\end{array}$} \\
\hline & & & & & & & & & & \\
\hline Dutkowski [13] 2015 & 5 & 25 & 22 & 50 & $13.0 \%$ & $0.32[0.10,0.98]$ & & & & \\
\hline Van Rijn [15] 2017 & 0 & 10 & 2 & 20 & $1.7 \%$ & $0.35[0.02,8.06]$ & & & & \\
\hline Patrono [16] 2019 & 8 & 25 & 17 & 50 & $15.8 \%$ & $0.91[0.33,2.54]$ & & & & \\
\hline Ravaioli [18] 2020 & 0 & 10 & 7 & 30 & $1.9 \%$ & $0.15[0.01,2.86]$ & 4 & & & \\
\hline Rayar [19] 2020 & 7 & 25 & 29 & 69 & $16.7 \%$ & $0.54[0.20,1.45]$ & & & - & \\
\hline Van Rijn [20] 2021 & 20 & 78 & 31 & 78 & $35.7 \%$ & $0.52[0.26,1.03]$ & & & & \\
\hline Subtotal $(95 \% \mathrm{Cl})$ & & 173 & & 297 & $84.9 \%$ & $0.52[0.34,0.81]$ & & & & \\
\hline Total events & 40 & & 108 & & & & & & & \\
\hline \multicolumn{11}{|c|}{$\begin{array}{l}\text { Heterogeneity: } \mathrm{Tau}^{2}=0.00 ; \mathrm{Chi}^{2}=2.66, \mathrm{df}=5(P=0.75) ; \mathrm{I}^{2}=0 \% \\
\text { Test for overall effect: } Z=2.89(P=0.004)\end{array}$} \\
\hline \multicolumn{11}{|l|}{ 1.1.2 Non-oxygenated } \\
\hline Guarrera [12] 2010 & 1 & 20 & 5 & 20 & $3.3 \%$ & $0.16[0.02,1.50]$ & & & - & \\
\hline Guarrera [14] 2015 & 6 & 31 & 9 & 30 & $11.8 \%$ & $0.56[0.17,1.83]$ & & & & \\
\hline Subtotal $(95 \% \mathrm{Cl})$ & & 51 & & 50 & $15.1 \%$ & $0.43[0.15,1.21]$ & & & & \\
\hline Total events & 7 & & 14 & & & & & & & \\
\hline \multicolumn{11}{|c|}{$\begin{array}{l}\text { Heterogeneity. } \mathrm{Tau}^{2}=0.00 ; \mathrm{Chi}^{2}=0.96, \mathrm{df}=1(P=0.33) ; \mathrm{I}^{2}=0 \% \\
\text { Test for overall effect: } Z=1.60(P=0.11)\end{array}$} \\
\hline Total $(95 \% \mathrm{Cl})$ & & 224 & & 347 & $100.0 \%$ & $0.51[0.34,0.76]$ & & & & \\
\hline Total events & 47 & & 122 & & & & & & & \\
\hline $\begin{array}{l}\text { Heterogeneity: } \mathrm{Tau}^{2}= \\
\text { Test for overall effect: } \\
\text { Test for subqroup dift }\end{array}$ & $\begin{array}{l}.00 ; \mathrm{Chi}^{2} \\
=3.28(\mathrm{P} \\
\text { rences: } \mathrm{C}\end{array}$ & $\begin{array}{l}=3.74 \\
=0.00 \\
h \mathrm{hi}^{2}=0\end{array}$ & $\begin{array}{l}d f=7(P \\
\text { 12. } d f=1\end{array}$ & $\begin{array}{l}=0.81 \\
(P=0\end{array}$ & $\begin{array}{l}: 1^{2}=0 \% \\
73) \cdot 1^{2}=0\end{array}$ & & 0.01 & $\begin{array}{c}1 \\
0.1 \\
\text { Favours HMP }\end{array}$ & $\begin{array}{c}10 \\
\text { Favours SCS }\end{array}$ & 100 \\
\hline
\end{tabular}

Fig. 2. Forest plot of studies comparing OR of EAD between HMP and SCS groups. OR, odds ratio; EAD, early allograft dysfunction; HMP, hypothermic perfusion; SCS, static cold storage; CI, confidence interval; M-H, Mantel-Haenszel.

largest MP study so far. In this multicenter RCT, a total of 222 patients (121 NMP vs. 101 SCS) successfully underwent LTx [24]. The main finding of the study was that grafts after NMP had 50\% lower levels of injury, measured by the peak level of serum AST within 7 days after transplantation. This result was achieved, despite a 50\% lower organ discard rate and 54\% longer mean preservation time in the NMP group. Furthermore, the authors observed a significantly lower EAD and postreperfusion syndrome rate in patients who received machine-perfused liver grafts. Although the short-term postoperative outcomes appear to favor NMP over conventional cold storage, long-term results, such as 12-month graft and patient survival, were similar between groups.

Another RCT was conducted in a single center by Ghinolfi et al. [25]. In this study, only donation after brain death (DBD) donors older than 70 years were enrolled. Results demonstrated only histological evidence of reduced graft injury in machine-perfused livers but did not show any clinical benefits of NMP. Complication rate, hospital stay, and 6-month graft and patient survival were similar in both groups.

The other 4 studies were case-matched and included both DBD and DCD donors [21-23, 26]. Ravikumar et al. [21] and Liu et al. [26] found significantly lower peak AST 


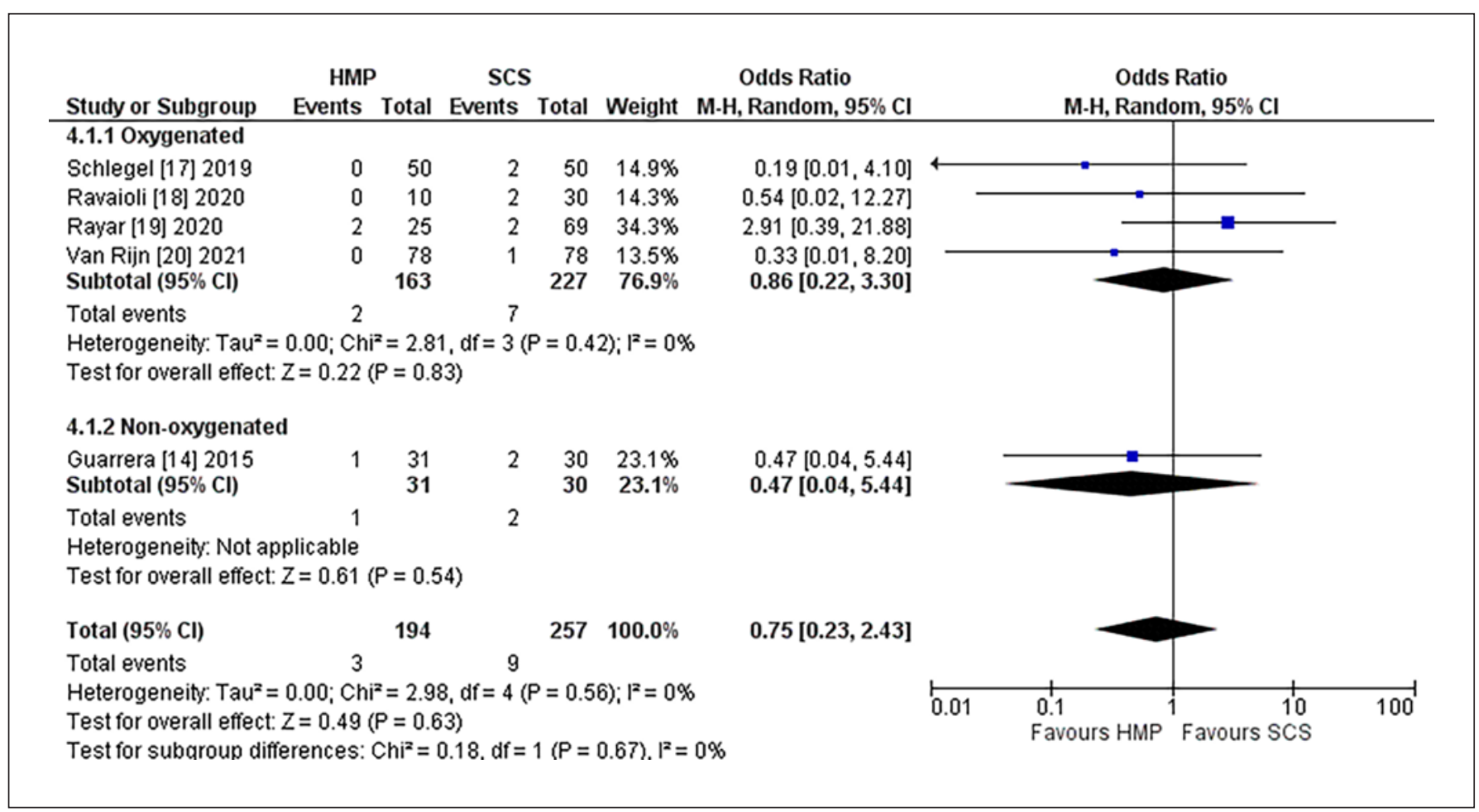

Fig. 3. Forest plot of studies comparing OR of PNF between HMP and SCS groups. OR, odds ratio; HMP, hypothermic perfusion; SCS, static cold storage; CI, confidence interval; M-H, Mantel-Haenszel; PNF, primary nonfunction.

levels in the NMP group patients. Additionally, Liu et al. [26] reported lower EAD rates in the NMP group. None of these studies showed any graft or patient survival benefits during their follow-up period.

Hypothermic Machine Perfusion

Early Allograft Dysfunction. The overall EAD rate in the HMP group was $20.1 \%$ (47/224) versus $35.2 \%$ $(122 / 347)$ in the SCS group. This difference was similar in both subgroups, and the overall effect was statistically significant (OR $0.51 ; 95 \%$ CI $0.34-0.76 ; p=0.001 ; I^{2}=0 \%$ ) (Fig. 2).

Primary Nonfunction. The overall effect in the graft PNF rate was not significant between groups (OR 0.75; 95\% CI 0.23-2.43; $p=0.63 ; I^{2}=0 \%$ ) (Fig. 3). The overall graft PNF rate in the HMP group was 1.5\% (3/194) compared to $3.5 \%(9 / 257)$ in the SCS group. Three studies, included in the meta-analysis, reported no cases of graft PNF $[12,15,16]$.

Biliary Complications. The overall total biliary complications (biliary strictures, leaks, and casts) rate was $29.3 \%$ (73/249) in the HMP group and $33.1 \%(115 / 347)$ in the SCS group, and there was a statistical significance in the overall effect between the groups (OR 0.63; 95\% CI 0.43$0.93 ; p=0.02 ; I^{2}=0 \%$ ) (Fig. 4 ). We further analyzed the influence of HMP on the rate of non-anastomotic biliary stricture between the groups. The rates in the HMP and
SCS were 6.6\% (12/183) and 17.9\% (39/218), respectively. This difference was statistically significant (OR $0.34 ; 95 \%$ CI $\left.0.17-0.67 ; p=0.002 ; I^{2}=0 \%\right)$, there were no differences between subgroups (Fig. 5). Three studies were not included in this analysis [14, 18, 19]. Ravaioli et al. [18] reported the rate of biliary strictures without specifying what type they were. In addition, Guarrera et al. [14] and Rayar et al. [19] observed no non-anastomotic biliary strictures in their study.

Mortality and Graft Loss within 12 Months. There was no significant difference in mortality rates between the groups (OR 0.57 ; 95\% CI $0.26-1.26 ; p=0.16 ; I^{2}=0 \%$ ), although the overall mortality rate in the SCS group was higher than that in the HMP group - 12.3\% (27/219) and $6.8 \%(10 / 146)$, respectively (Fig. 6). Similar results were seen in the graft loss rate analysis. The findings did not reach statistical significance (OR 0.63 ; 95\% CI 0.33-1.22; $\left.p=0.17 ; I^{2}=0 \%\right)$, but the graft loss rate was higher in the SCS $(17.8 \%$ [39/219]) than $11.0 \%$ in the HMP group (16/146) (Fig. 7). We did not include 2 studies in this analysis. Patrono et al. [16] did not report these data for the SCS group. Van Rijn et al. [20] report only 6-month patient survival and graft loss; thus, we did not include it in this analysis. 


\begin{tabular}{|c|c|c|c|c|c|c|c|c|c|c|}
\hline Study or Subgroup & $\begin{array}{l}\text { HMP } \\
\text { Events }\end{array}$ & & $\begin{array}{l}\text { SCS } \\
\text { Events }\end{array}$ & Total & Weight & $\begin{array}{l}\text { Odds Ratio } \\
\text { M-H, Random, } 95 \% \mathrm{Cl}\end{array}$ & \multicolumn{4}{|c|}{$\begin{array}{c}\text { Odds Ratio } \\
\text { M-H, Random, } 95 \% \mathrm{Cl}\end{array}$} \\
\hline Van Rijn [15] 2017 & 4 & 10 & 11 & 20 & $6.3 \%$ & $0.55[0.12,2.55]$ & & & & \\
\hline Patrono [16] 2019 & 6 & 25 & 9 & 50 & $10.9 \%$ & $1.44[0.45,4.62]$ & & & . & \\
\hline Schlegel [17] 2019 & 20 & 50 & 23 & 50 & $23.7 \%$ & $0.78[0.35,1.73]$ & & $\rightarrow$ & - & \\
\hline Ravaioli [18] 2020 & 1 & 10 & 3 & 30 & $2.6 \%$ & $1.00[0.09,10.87]$ & & & & \\
\hline Rayar [19] 2020 & 2 & 25 & 8 & 69 & $5.7 \%$ & $0.66[0.13,3.36]$ & & & & \\
\hline $\begin{array}{l}\text { Van Rijn }[20] 2021 \\
\text { Subtotal }(95 \% \mathrm{Cl})\end{array}$ & 34 & $\begin{array}{r}78 \\
198\end{array}$ & 44 & $\begin{array}{r}78 \\
297\end{array}$ & $\begin{array}{l}37.2 \% \\
86.4 \%\end{array}$ & $\begin{array}{l}0.60[0.32,1.12] \\
0.73[0.48,1.11]\end{array}$ & & & & \\
\hline \multicolumn{11}{|c|}{$\begin{array}{l}\text { Heterogeneity: } \operatorname{Tau}^{2}=0.00 ; \mathrm{Chi}^{2}=1.93, \mathrm{df}=5(P=0.86) ; \mathrm{I}^{2}=0 \% \\
\text { Test for overall effect: } Z=1.48(P=0.14)\end{array}$} \\
\hline \multicolumn{11}{|l|}{ 3.1.2 Non-oxygenated } \\
\hline Guarrera [12] 2010 & 2 & 20 & 4 & 20 & $4.5 \%$ & $0.44[0.07,2.76]$ & & & & \\
\hline $\begin{array}{l}\text { Guarrera [14] } 2015 \\
\text { Subtotal }(95 \% \mathrm{Cl})\end{array}$ & 4 & $\begin{array}{l}31 \\
51\end{array}$ & 13 & $\begin{array}{l}30 \\
50\end{array}$ & $\begin{array}{r}9.2 \% \\
13.6 \%\end{array}$ & $\begin{array}{l}0.19[0.05,0.69] \\
0.25[0.09,0.72]\end{array}$ & & & & \\
\hline \multicolumn{11}{|c|}{$\begin{array}{l}\text { Heterogeneity: } \mathrm{Tau}^{2}=0.00 ; \mathrm{Chi}^{2}=0.53, \mathrm{df}=1(P=0.46) ; \mathrm{l}^{2}=0 \% \\
\text { Test for overall effect: } Z=2.57(P=0.01)\end{array}$} \\
\hline Total $(95 \% \mathrm{Cl})$ & & 249 & & 347 & $100.0 \%$ & $0.63[0.43,0.93]$ & & & & \\
\hline Total events & 73 & & 115 & & & & & & & \\
\hline $\begin{array}{l}\text { Heterogeneity. Tau } \\
\text { Test for overall effect } \\
\text { Test for subaroup di }\end{array}$ & $\begin{array}{l}0.00 ; \mathrm{Chi} \\
\mathrm{Z}=2.33 \\
\text { erences: }\end{array}$ & $\begin{array}{l}\mathrm{i}^{2}=5.8 \\
(\mathrm{P}=0.0 \\
\mathrm{Chi}^{2}=\end{array}$ & $\begin{array}{l}5, d f=7( \\
2 \text { 2) } \\
3.38 . d f=\end{array}$ & $\begin{array}{l}P=0.5 \\
1(P=\end{array}$ & $\begin{array}{l}6) ; 1^{2}=09 \\
0.07) \cdot 1^{2}=\end{array}$ & $70.4 \%$ & 0.01 & $\begin{array}{l}0.1 \\
\text { Favours HMP }\end{array}$ & $\begin{array}{r}10 \\
\text { Favours SCS }\end{array}$ & 100 \\
\hline
\end{tabular}

Fig. 4. Forest plot of studies comparing OR of total biliary complications between HMP and SCS groups. OR, odds ratio; HMP, hypothermic perfusion; SCS, static cold storage; $\mathrm{CI}$, confidence interval; $\mathrm{M}-\mathrm{H}$, Mantel-Haenszel.

\begin{tabular}{|c|c|c|c|c|c|c|c|c|c|c|}
\hline \multirow{2}{*}{$\frac{\text { Study or Subgroup }}{3.2 .1 \text { Oxygenated }}$} & $\begin{array}{l}\text { HMP } \\
\text { Events }\end{array}$ & P & \multicolumn{2}{|c|}{$\operatorname{scs}$} & Weight & $\begin{array}{l}\text { Odds Ratio } \\
\text { M-H, Random, } 95 \% \mathrm{Cl}\end{array}$ & \multicolumn{4}{|c|}{$\begin{array}{c}\text { Odds Ratio } \\
\text { M-H, Random, } 95 \% \mathrm{Cl}\end{array}$} \\
\hline & & & & & & & & & & \\
\hline Van Rijn [15] 2017 & 1 & 10 & 7 & 20 & $9.1 \%$ & $0.21[0.02,1.98]$ & & & - & \\
\hline Patrono [16] 2019 & 2 & 25 & 4 & 50 & $14.8 \%$ & $1.00[0.17,5.87]$ & & & & \\
\hline Schlegel [17] 2019 & 4 & 50 & 11 & 50 & $31.0 \%$ & $0.31[0.09,1.05]$ & & & & \\
\hline $\begin{array}{l}\text { Van Rijn [20] } 2021 \\
\text { Subtotal }(95 \% \mathrm{CI})\end{array}$ & 5 & $\begin{array}{r}78 \\
163\end{array}$ & 14 & $\begin{array}{r}78 \\
198\end{array}$ & $\begin{array}{l}40.1 \% \\
95.0 \%\end{array}$ & $\begin{array}{l}0.31[0.11,0.92] \\
0.36[0.18,0.72]\end{array}$ & & & & \\
\hline \multicolumn{11}{|c|}{$\begin{array}{l}\text { Heterogeneity: } \text { Tau }^{2}=0.00 ; \mathrm{Chi}^{2}=1.64, d f=3(P=0.65) ; I^{2}=0 \% \\
\text { Test for overall effect: } Z=2.88(P=0.004)\end{array}$} \\
\hline \multicolumn{11}{|l|}{ 3.2.2 Non-oxygenated } \\
\hline $\begin{array}{l}\text { Guarrera [12] } 2010 \\
\text { Subtotal }(95 \% \mathrm{Cl})\end{array}$ & 0 & $\begin{array}{l}20 \\
20\end{array}$ & 3 & $\begin{array}{l}20 \\
20\end{array}$ & $5.0 \%$ & $\begin{array}{l}0.12[0.01,2.53] \\
0.12[0.01,2.53]\end{array}$ & & & & \\
\hline \multicolumn{11}{|c|}{$\begin{array}{l}\text { Heterogeneity: Not applicable } \\
\text { Test for overall effect: } Z=1.36(P=0.17)\end{array}$} \\
\hline Total $(95 \% \mathrm{Cl})$ & & 183 & & 218 & $100.0 \%$ & $0.34[0.17,0.67]$ & & & & \\
\hline Total events & 12 & & 39 & & & & & & & \\
\hline $\begin{array}{l}\text { Heterogeneity. Tau } \\
\text { Test for overall effect } \\
\text { Test for subaroup di }\end{array}$ & $\begin{array}{l}0.00 ; \mathrm{Chi}^{-} \\
\mathrm{Z}=3.11 \\
\text { erences: }\end{array}$ & 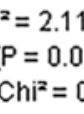 & $\begin{array}{l}\text { 1. } d f=4(f \\
02) \\
0.46 . d f=\end{array}$ & $\begin{array}{l}P=0.7 \\
1(P=\end{array}$ & $\begin{array}{l}2) ; 1^{2}=0 \% \\
0.50) \cdot 1^{2}=\end{array}$ & $0 \%$ & $\frac{1}{0.01}$ & $\begin{array}{c}0.1 \\
\text { Favours HMP }\end{array}$ & $\begin{array}{c}10 \\
\text { Favours SCS }\end{array}$ & 100 \\
\hline
\end{tabular}

Fig. 5. Forest plot of studies comparing OR of non-anastomotic biliary strictures between HMP and SCS groups. OR, odds ratio; HMP, hypothermic perfusion; SCS, static cold storage; CI, confidence interval; M-H, MantelHaenszel. 
Study or Subgroup

HMP SCS

Odds Ratio

Odds Ratio

Events Total Events Total Weight $\mathrm{M} \cdot \mathrm{H}, \mathrm{Random}, 95 \% \mathrm{Cl}$

M.H, Random, $95 \% \mathrm{Cl}$

2.1.1 Oxygenated

Van Rijn [15] 2017

Schlegel [17] 2019

Ravaioli [18] 2020

Rayar [19] 2020

Subtotal $(95 \% \mathrm{CI})$

Total events

$\begin{array}{lllll}0 & 10 & 3 & 20 & 6.6 \%\end{array}$

$\begin{array}{lllll}1 & 50 & 7 & 50 & 13.6 \%\end{array}$

$\begin{array}{lllll}0 & 10 & 3 & 30 & 6.7 \%\end{array}$

$\begin{array}{lllll}2 & 25 & 6 & 69 & 22.3 \%\end{array}$

$95 \quad 169 \quad 49.2 \%$

3

Heterogeneity: $\mathrm{Tau}^{2}=0.00 ; \mathrm{Chi}^{2}=2.23, \mathrm{df}=3(\mathrm{P}=0.53) ; \mathrm{I}^{2}=0 \%$

Test for overall effect: $Z=1.65(P=0.10)$

2.1.2 Non-oxygenated

$\begin{array}{lllllll}\text { Guarrera [12] 2010 } & 2 & 20 & 2 & 20 & 14.6 \% & 1.00[0.13,7.89] \\ \text { Guarrera [14] 2015 } & 5 & 31 & 6 & 30 & 36.2 \% & 0.77[0.21,2.85] \\ \text { Subtotal (95\% CI) } & & \mathbf{5 1} & & \mathbf{5 0} & \mathbf{5 0 . 8 \%} & \mathbf{0 . 8 3}[\mathbf{0 . 2 7}, \mathbf{2 . 5 1}\end{array}$

$7 \quad 8$

Heterogeneity. Tau $^{2}=0.00 ; \mathrm{Chi}^{2}=0.04, \mathrm{df}=1(\mathrm{P}=0.83) ; \mathrm{I}^{2}=0 \%$

Test for overall effect: $Z=0.33(P=0.74)$

$\begin{array}{lllll}\text { Total }(95 \% \mathrm{Cl}) & 146 & 219 & 100.0 \% & 0.57[0.26,1.26]\end{array}$

Total events 27

Heterogeneity: Tau $^{2}=0.00 ; \mathrm{Chi}^{2}=3.21, \mathrm{df}=5(\mathrm{P}=0.67) ; \mathrm{I}^{2}=0 \%$

Test for overall effect: $Z=1.39(P=0.16)$

Test for subaroup differences: $\mathrm{Chi}^{2}=0.88 . \mathrm{df}=1(\mathrm{P}=0.35) . \mathrm{I}^{2}=0 \%$

$0.24[0.01,5.08]$

$0.13[0.01,1.06]$

$0.37[0.02,7.88]$

$0.91[0.17,4.85]$

$0.39[0.13,1.20]$

$0.83[0.27,2.51]$
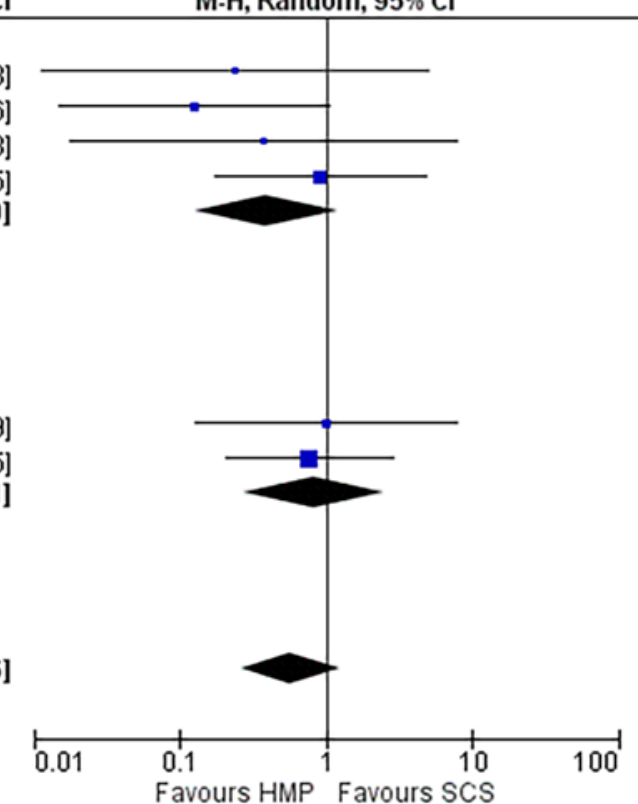

Fig. 6. Forest plot of studies comparing OR of graft loss within 12 months between HMP and SCS groups. OR, odds ratio; HMP, hypothermic perfusion; SCS, static cold storage; $\mathrm{CI}$, confidence interval; $\mathrm{M}-\mathrm{H}$, Mantel-Haenszel.

\begin{tabular}{|c|c|c|c|c|c|c|c|c|c|c|}
\hline \multirow{2}{*}{$\frac{\text { Study or Subgroup }}{2.2 .1 \text { Oxygenated }}$} & \multirow[t]{2}{*}{$\begin{array}{r}\text { HMF } \\
\text { Events } \\
\end{array}$} & & \multicolumn{2}{|c|}{ SCS } & \multirow[t]{2}{*}{ Weight } & \multirow[t]{2}{*}{$\begin{array}{c}\text { Odds Ratio } \\
\text { M-H, Random, } 95 \% \mathrm{Cl}\end{array}$} & \multicolumn{4}{|c|}{$\begin{array}{c}\text { Odds Ratio } \\
\text { M-H, Random, } 95 \% \mathrm{Cl}\end{array}$} \\
\hline & & & & & & & & & & \\
\hline Van Rijn [15] 2017 & 0 & 10 & 9 & 20 & $4.9 \%$ & $0.06[0.00,1.12]$ & $\longleftarrow$ & & & \\
\hline Schlegel [1\}] 2019 & 5 & 50 & 9 & 50 & $31.2 \%$ & $0.51[0.16,1.63]$ & & & & \\
\hline Ravaioli [18] 2020 & 0 & 10 & 3 & 30 & $4.6 \%$ & $0.37[0.02,7.88]$ & & & & \\
\hline $\begin{array}{l}\text { Rayar }[19] 2020 \\
\text { Subtotal }(95 \% \mathrm{Cl})\end{array}$ & 3 & $\begin{array}{l}25 \\
95\end{array}$ & 10 & $\begin{array}{r}69 \\
169\end{array}$ & $\begin{array}{l}22.5 \% \\
63.1 \%\end{array}$ & $\begin{array}{l}0.80[0.20,3.20] \\
0.49[0.22,1.13]\end{array}$ & & & & \\
\hline \multicolumn{11}{|c|}{$\begin{array}{l}\text { Heterogeneity: } \text { Tau }^{2}=0.00 ; \mathrm{Chi}^{2}=2.65, \mathrm{df}=3(\mathrm{P}=0.45) ; \mathrm{I}^{2}=0 \% \\
\text { Test for overall effect: } Z=1.68(P=0.09)\end{array}$} \\
\hline \multicolumn{11}{|c|}{ 2.2.2 Non-oxygenated } \\
\hline Guarrera [12] 2010 & 2 & 20 & 2 & 20 & $10.0 \%$ & $1.00[0.13,7.89]$ & & & & \\
\hline $\begin{array}{l}\text { Guarrera [14] } 2015 \\
\text { Subtotal }(95 \% \mathrm{Cl})\end{array}$ & 6 & $\begin{array}{l}31 \\
51\end{array}$ & 6 & $\begin{array}{l}30 \\
50\end{array}$ & $\begin{array}{l}26.9 \% \\
36.9 \%\end{array}$ & $\begin{array}{l}0.96[0.27,3.39] \\
0.97[0.33,2.85]\end{array}$ & & & & \\
\hline \multicolumn{11}{|c|}{$\begin{array}{l}\text { Heterogeneity: } \text { Tau }^{2}=0.00 ; \mathrm{Chi}^{2}=0.00, \mathrm{df}=1(\mathrm{P}=0.97) ; \mathrm{I}^{2}=0 \% \\
\text { Test for overall effect: } Z=0.05(P=0.96)\end{array}$} \\
\hline Total $(95 \% \mathrm{Cl})$ & & 146 & & 219 & $100.0 \%$ & $0.63[0.33,1.22]$ & & & & \\
\hline Total events & 16 & & 39 & & & & & & & \\
\hline $\begin{array}{l}\text { Heterogeneity: Tau } \\
\text { Test for overall effect } \\
\text { Test for subqroun di }\end{array}$ & $\begin{array}{l}0.00 ; \text { Chi } \\
Z=1.37 \\
\text { erences: }\end{array}$ & $\begin{array}{l}\mathrm{l}^{2}=3.6 \\
\mathrm{P}=0.1 \\
\mathrm{Chi}^{2}=\end{array}$ & $\begin{array}{l}2, d f=5( \\
\text { 7) } \\
0.95 . d f=\end{array}$ & $\begin{array}{l}P=0.6 \\
1(P=\end{array}$ & $\begin{array}{l}1) ; I^{2}=09 \\
0.33) \cdot I^{2}=\end{array}$ & & $\stackrel{\longmapsto}{\circ .01}$ & $\begin{array}{l}0.1 \\
\text { Favours HMP }\end{array}$ & Favours SCS & 100 \\
\hline
\end{tabular}

Fig. 7. Forest plot of studies comparing OR of mortality within 12 months between HMP and SCS groups. OR, odds ratio; HMP, hypothermic perfusion; SCS, static cold storage. 


\section{Discussion/Conclusion}

In this systematic review and meta-analysis, we overviewed the potential effects of MP on liver grafts in a clinical LTx setting. The primary aim was to perform quantitative analysis on both HMP and NMP studies. We included 6 studies that investigated the benefits of NMP, 2 of them were RCTs and the other 4 were observational studies. Due to high heterogeneity in study design and partly to technical variances of perfusion between studies, methodologically, we could not pool all studies into 1 analysis; thus, we decided only to present a qualitative analysis of studies examining NMP. The included studies revealed the potential of NMP to reduce graft injury and lower the liver graft discard rate, which allows broader utilization of liver from DCD [21-26].

From the meta-analysis performed on HMP studies, we concluded that the use of HMP reduces the rate of EAD, total biliary complications, and non-anastomotic biliary strictures compared to SCS. Although the 12-month graft and patient survival had a tendency to favor HMP, these long-term outcomes failed to reach statistical significance.

Currently, there is an ongoing discussion and criticism toward studies evaluating the role of MP in LTx $[27,28]$. The main argument is that such studies should focus more on clinically relevant outcomes, for instance, patient survival, graft loss or ischemic cholangiopathy and not on surrogate outcomes, such as peak serum aminotransferase levels. There are studies showing that peak postoperative AST levels may have some value in predicting long-term postoperative outcomes [29]. However, it should be noted that they do not take into account the washout phenomena that occur, when liver is flushed with a large amount of preservation solution or reperfused and oxygenated during MP. Different metabolites, cytokines, and transaminases accumulate in the perfusion system but not in recipient right after the transplantation [30-32]. Thus, such predictive models cannot be used to accurately evaluate the effects of MP on the quality of the liver.

Currently, there is a lack of literature quantitatively analyzing the benefits of MP. Porcine models were a crucial part in bringing MP studies to the clinics; thus, metaanalyses by Bian et al. and Nostedt et al. try to summarize the effects of NMP on porcine liver [33,34]. Both metaanalyses concluded that NMP is superior to SCS in preserving the liver architecture and function; unfortunately, only short-term outcomes, such as the postoperative levels of ALT and AST or bile production, were available for analysis. The first meta-analysis on human studies was conducted by Zhang et al. [35], and it found that HMP could significantly reduce the incidence of EAD and biliary complications. However, this meta-analysis includes overlapping studies possibly magnifying the true protective effects of HMP. A recent meta-analysis by Jia et al. [36] overcomes this issue and analyzes both HMP and NMP against SCS. They concluded that the incidence of EAD and biliary complications were significantly lower in recipients with MP preservation. Although they performed a subgroup analysis with HMP and NMP, a meta-analysis trying to draw conclusions about the whole clinical MP field is pointless due to enormous heterogeneity of the studies and completely different underlying aims and mechanisms of both MP types [7].

Our study has some limitations, which should be considered. First of all, most of the included studies were nonrandomized; however, they all were case-matched for at least donor age, type (DCD and DBD), and recipient's MELD score. Furthermore, most of them showed moderate risk of bias when assessed with the ROBINS-I tool. To be noted, studies by Dutkowski et al. [13] and Schlegel et al. [17] were evaluated as having a severe risk of bias, due to differences of immunosuppression therapy between the HMP and SCS groups. In this case, we tested the robustness of our data by conducting a sensitivity analysis, and we did not see significant changes in our results. Second, different perfusion settings were applied in included studies. We tried to partly overcome this limitation by performing a subgroup analysis according to whether additional oxygenation was used or not during MP.

These previously mentioned MP types are technically very different with their own specific advantages and disadvantages. NMP simulates normal liver cell metabolism, which allows for better organ viability assessment $[7,37]$. Furthermore, NMP can be utilized for organ repair as different therapeutic agents are currently being investigated [37]. On the other hand, user or device error when using NMP has serious consequences, quite often leading to graft loss. The aforementioned drawback is not that meaningful in the use of HMP as the organ is in a reduced metabolism state. Moreover, the lower initial cost and promising first results make HMP a strong contender to NMP. There is an ongoing trial (NCT04644744) directly evaluating HOPE versus NMP in LTx, which may further highlight the drawbacks and benefits of these MP types.

This research area still lacks high-quality data from randomized trials. Currently, there are only 2 published RCTs, and both of them analyze NMP $[24,25]$. The results from several currently ongoing or completed RCTs examining the use of HMP are eagerly awaited (NCT01317342, NCT03484455, NCT03837197, NCT03929523, and NCT03124641).

The current critical liver donation situation prompts the use of ECD or DCD donors with inferior overall re- 
sults [38]. The routine use of MP systems could not only increase the quality of these suboptimal liver grafts but also broaden the potential donor pool helping to narrow the gap between organ availability and demand [7].

In conclusion, our systematic review and meta-analysis revealed that the use of HMP reduces the rate of EAD and non-anastomotic biliary strictures compared to SCS. Additionally, the currently available literature shows the potential of NMP to reduce graft injury and lower liver graft discard rate. These findings may provide guidance in choosing the optimal liver preservation method before transplantation.

\section{Statement of Ethics}

The authors assert that all procedures contributing to this work comply with the ethical standards of the relevant national and institutional committees on human experimentation and with the Helsinki Declaration of 1975, as revised in 2008.

\section{Conflict of Interest Statement}

The authors have no conflicts of interest to declare.

\section{Funding Sources}

This research did not receive any specific grant from funding agencies in the public, commercial, or not-for-profit sectors.

\section{Author Contributions}

P.Sc., P.St., B.L., and K.S. contributed to conceptualization; M.J., L.J., P.Sc., and P.St contributed to methodology; M.J. and L.J. contributed to software; P.Sc. and P.St., and B.L. contributed to validation; M.J. and L.J. contributed to formal analysis; M.J., L.J., P.Sc., P.St., B.L., and K.S. contributed to investigation; P.Sc., P.St., and B.L. contributed to resources; M.J. and L.J. contributed to data curation; M.J. and L.J. contributed to writing - original draft preparation; P.Sc., P.St., B.L., and K.S. contributed to writing - review and editing; M.J. and L.J. contributed to visualization; P.Sc., P.St., B.L., and K.S. contributed to supervision; and P.Sc., P.St., and B.L. contributed to project administration.

\section{Data Availability Statement}

Data are available on reasonable request. All data relevant to the study are included in the article or uploaded as online supplementary information. De-identified data, which underlie the results reported in this article, will be shared with third parties after written request to the corresponding author describing intention of data usage and full affiliation of the requesting organization.

\section{References}

1 Starzl TE, Marchioro TL, Vonkaulla KN, Hermann G, Brittain RS, Waddell WR. Homotransplantation of the liver in humans. Surg Gynecol Obstet. 1963 Dec;117:659-76.

2 Jain A, Reyes J, Kashyap R, Dodson SF, Demetris AJ, Ruppert K, et al. Long-term survival after liver transplantation in 4,000 consecutive patients at a single center. Ann Surg. 2000 Oct;232(4):490-500.

3 Belzer FO, Southard JH. Principles of solidorgan preservation by cold storage. Transplantation. 1988 Apr;45(4):673-6.

4 Vogel T, Brockmann JG, Coussios C, Friend PJ. The role of normothermic extracorporeal perfusion in minimizing ischemia reperfusion injury. Transplant Rev. 2012 Apr;26(2): 156-62.

5 Kvietkauskas M, Leber B, Strupas K, Stiegler P, Schemmer P. Machine perfusion of extended criteria donor organs: immunological aspects. Front Immunol. 2020 Feb 27;11:192.

6 Henry SD, Nachber E, Tulipan J, Stone J, Bae C, Reznik L, et al. Hypothermic machine preservation reduces molecular markers of ische$\mathrm{mia} /$ reperfusion injury in human liver transplantation: liver machine perfusion reduces ischemia/reperfusion injury. Am J Transplant. 2012 Sep;12(9):2477-86.

7 Czigany Z, Lurje I, Tolba RH, Neumann UP, Tacke F, Lurje G. Machine perfusion for liver transplantation in the era of marginal organsnew kids on the block. Liver Int. 2019 Feb; 39(2):228-49.

8 Karangwa SA, Dutkowski P, Fontes P, Friend PJ, Guarrera JV, Markmann JF, et al. Machine perfusion of donor livers for transplantation: a proposal for standardized nomenclature and reporting guidelines. Am J Transplant. 2016 Oct;16(10):2932-42.

9 Sterne JA, Hernán MA, Reeves BC, Savović J, Berkman ND, Viswanathan M, et al. ROBINS-I: a tool for assessing risk of bias in nonrandomised studies of interventions. BMJ. 2016 Oct 12;355:i4919.

10 Sterne JAC, Savović J, Page MJ, Elbers RG, Blencowe NS, Boutron I, et al. RoB 2: a revised tool for assessing risk of bias in randomised trials. BMJ. 2019 Aug 28;366:14898.

11 Cochrane handbook for systematic reviews of interventions [Internet]. [cited 2020 Jun 12]. Available from: https://www.training.cochrane.org/handbook/current.

12 Guarrera JV, Henry SD, Samstein B, OdehRamadan R, Kinkhabwala M, Goldstein MJ, et al. Hypothermic machine preservation in human liver transplantation: the first clinical series. Am J Transplant. 2010 Feb;10(2):372-81.

13 Dutkowski P, Polak WG, Muiesan P, Schlegel A, Verhoeven CJ, Scalera I, et al. First comparison of hypothermic oxygenated perfusion versus static cold storage of human donation after cardiac death liver transplants: an international-matched case analysis. Ann Surg. 2015 Nov;262(5):764-1.

14 Guarrera JV, Henry SD, Samstein B, Reznik E, Musat C, Lukose TI, et al. Hypothermic machine preservation facilitates successful transplantation of "orphan" extended criteria donor livers: machine preservation of ECD livers. Am J Transplant. 2015 Jan;15(1):161-9.
15 van Rijn R, Karimian N, Matton APM, Burlage LC, Westerkamp AC, van den Berg AP, et al. Dual hypothermic oxygenated machine perfusion in liver transplants donated after circulatory death: dual hypothermic oxygenated machine perfusion in liver transplantation. Br J Surg. 2017 Jun;104(7):907-17.

16 Patrono D, Surra A, Catalano G, Rizza G, Berchialla P, Martini S, et al. Hypothermic oxygenated machine perfusion of liver grafts from brain-dead donors. Sci Rep. 2019 Dec; 9(1):9337.

17 Schlegel A, Muller X, Kalisvaart M, Muellhaupt B, Perera MTPR, Isaac JR, et al. Outcomes of DCD liver transplantation using organs treated by hypothermic oxygenated perfusion before implantation. J Hepatol. 2019 Jan;70(1):50-7.

18 Ravaioli M, De Pace V, Angeletti A, Comai G, Vasuri F, Baldassarre M, et al. Hypothermic oxygenated new machine perfusion system in liver and kidney transplantation of extended criteria donors:first Italian clinical trial. Sci Rep. 2020 Dec;10(1):6063.

19 Rayar M, Beaurepaire J, Bajeux E, Hamonic S, Renard T, Locher C, et al. Hypothermic oxygenated perfusion(HOPE) improves ECD liver graft function and reduces duration of hospitalisation without extra cost: the PERPHO Study. Liver Transpl. 2021 Feb;27(3):349-62.

20 van Rijn R, Schurink IJ, de Vries Y, van den Berg AP, Cortes Cerisuelo M, Darwish Murad $\mathrm{S}$, et al. Hypothermic machine perfusion in liver transplantation - a randomized trial. N Engl J Med. 2021 Apr 15;384(15):1391-401. 
21 Ravikumar R, Jassem W, Mergental H, Heaton N, Mirza D, Perera MT, et al. Liver transplantation after ex vivo normothermic machine preservation: a phase 1 (first-in-man) clinical trial. Am J Transplant. 2016 Jun;16(6): 1779-87.

22 Selzner M, Goldaracena N, Echeverri J, Kaths JM, Linares I, Selzner N, et al. Normothermic ex vivo liver perfusion using steen solution as perfusate for human liver transplantation: first North American results. Liver Transpl. 2016 Nov;22(11):1501-8.

23 Bral M, Gala-Lopez B, Bigam D, Kneteman N, Malcolm A, Livingstone S, et al. Preliminary single-center Canadian experience of human normothermic ex vivo liver perfusion: results of a clinical trial. Am J Transplant. 2017 Apr; 17(4):1071-80.

24 Nasralla D, Coussios CC, Mergental H, Akhtar MZ, Butler AJ, Ceresa CDL, et al. A randomized trial of normothermic preservation in liver transplantation. Nature. 2018; 557(7703):50-6.

25 Ghinolfi D, Rreka E, De Tata V, Franzini M, Pezzati D, Fierabracci V, et al. Pilot, open, randomized, prospective trial for normothermic machine perfusion evaluation in liver transplantation from older donors: liver transplantation. Liver Transpl. 2019 Mar; 25(3):436-49.

26 Liu Q, Hassan A, Pezzati D, Soliman B, Lomaglio L, Grady P, et al. Ex situ liver machine perfusion: the impact of fresh frozen plasma. Liver Transpl. 2020 Feb;26(2):215-26.
27 Dutkowski P, Guarrera JV, de Jonge J, Martins PN, Porte RJ, Clavien PA. Evolving trends in machine perfusion for liver transplantation. Gastroenterology. 2019 May;156(6): 1542-7.

28 Yeh $\mathrm{H}$, Uygun $\mathrm{K}$. Increasing donor liver utilization through machine perfusion. Hepatology. 2019 Jul;70(1):431-3.

29 Robertson FP, Bessell PR, Diaz-Nieto R, Thomas N, Rolando N, Fuller B, et al. High serum aspartate transaminase levels on day 3 postliver transplantation correlates with graft and patient survival and would be a valid surrogate for outcome in liver transplantation clinical trials. Transpl Int. 2016 Mar;29(3): 323-30.

30 Feng S, Goodrich NP, Bragg-Gresham JL, Dykstra DM, Punch JD, DebRoy MA, et al. Characteristics associated with liver graft failure: the concept of a donor risk index. Am J Transplant. 2006 Apr;6(4):783-90.

31 Braat AE, Blok JJ, Putter H, Adam R, Burroughs AK, Rahmel AO, et al. The Eurotransplant donor risk index in liver transplantation: ET-DRI. Am J Transplant. 2012 Oct; 12(10):2789-96.

32 Kaltenbach MG, Harhay MO, Abt PL, Goldberg DS. Trends in deceased donor liver enzymes prior to transplant: The impact on graft selection and outcomes. Am J Transplant. 2020 Jan;20(1):213-9.
33 Bian S, Zhu Z, Sun L, Wei L, Qu W, Zeng Z, et al. Normothermic Machine Perfusion versus Cold Storage of Liver in Pig Model: A Meta-Analysis. Ann Transplant. 2018;23:197206. 29567935.

34 Nostedt JJ, Skubleny DT, Shapiro AMJ, Campbell S, Freed DH, Bigam DL. Normothermic Ex Vivo Machine Perfusion for Liver Grafts Recovered from Donors after Circulatory Death: A Systematic Review and MetaAnalysis. HPB Surg. 2018. 29849531.

35 Zhang Y, Zhang Y, Zhang M, Ma Z, Wu S Hypothermic machine perfusion reduces the incidences of early allograft dysfunction and biliary complications and improves 1-year graft survival after human liver transplantation: a meta-analysis. Medicine. 2019 Jun; 98(23):e16033.

36 Jia J, Nie Y, Li J, Xie H, Zhou L, Yu J, et al. A systematic review and meta-analysis of machine perfusion vs. static cold storage of liver allografts on liver transplantation outcomes: the future direction of graft preservation. Front Med. 2020 May 12; 7:135.

37 Martins PN, Buchwald JE, Mergental H, Vargas L, Quintini C. The role of normothermic machine perfusion in liver transplantation. Int J Surg. 2020 Oct;82S:52-60.

38 Nardo B, Masetti M, Urbani L, Caraceni P, Montalti R, Filipponi F, et al. Liver transplantation from donors aged 80 years and over: pushing the limit. Am J Transplant. 2004; 4(7):1139-47. 